\title{
A future observational plan of dust particles around the Moon by LDM (Lunar Dust Monitor) onboard the orbiter of the next Japanese lunar mission
}

\author{
Masanori Kobayashi ${ }^{1}$, Hideo Ohashi ${ }^{2}$, Sho Sasaki $^{3}$, Hiromi Shibata ${ }^{4}$, Takeo Iwai ${ }^{5}$, \\ Masayuki Fujii ${ }^{6}$, Ken-ichi Nogami ${ }^{7}$, Hiroshi Kimura ${ }^{8}$, Maki H. Nakamura ${ }^{9}$, \\ Takayuki Hirai $^{10}$, Ralf Srama ${ }^{11}$, and Eberhard Grün ${ }^{11,12}$ \\ ${ }^{1}$ Planetary Exploration Research Center, Chiba Institute of Technology, Narashino, Chiba 275-0016, Japan \\ ${ }^{2}$ Department of Ocean Sciences, Tokyo University Marine Science and Technology, Shinagawa, Tokyo 108-8477, Japan \\ ${ }^{3}$ RISE project, National Astronomical Observatory of Japan, Mizusawa, Iwate 023-0861, Japan \\ ${ }^{4}$ Graduate School of Engineering, Kyoto University, Sakyo, Kyoto 606-8501, Japan \\ ${ }^{5}$ High Fluence Irradiation Facility, University of Tokyo, Tokai, Ibaraki 319-1188, Japan \\ ${ }^{6}$ Advanced Research Institute for Science and Engineering, Waseda University, Shinjuku, Tokyo 169-8555, Japan \\ ${ }^{7}$ Dokkyo Medical University, Shimotsuga, Tochigi 321-0293, Japan \\ ${ }^{8}$ Center for Planetary Science (CPS), Kobe 650-0047, Japan \\ ${ }^{9}$ Graduate School of Frontier Sciences, University of Tokyo, Kashiwa, Chiba 277-8582, Japan \\ ${ }^{10}$ School of Physical Sciences, Graduate University Advanced Studies, Hayama, Kanagawa 240-0193, Japan \\ ${ }^{11}$ Max Planck Institute for Nuclear Physics, D-69117 Heidelberg, Germany \\ ${ }^{12}$ Laboratory for Atmospheric and Space Physics, University of Colorado, Boulder, CO 80303-7814, USA
}

(Received October 29, 2010; Revised May 25, 2011; Accepted May 27, 2011; Online published February 2, 2012)

\begin{abstract}
This paper describes our future observation of the dust environment around the Moon by the Lunar Dust Monitor (LDM) to increase our knowledge regarding how the dust inflow and outflow contribute to lunar surface materials. Dust observation in lunar orbit is of great significance to better understand the source of supply of lunar materials, the evolution of lunar regolith, ejecta escaped from the Moon's gravitational sphere, and the inflow dust related to meteor streams. Although there have been several past missions of dust observation around the Moon, the origins of the observed dust particles in those missions could not be identified due to low statistics of dust flux or low accuracy of determining their arrival directions. To quantitatively study dust particles around the Moon, we need further data for improved statistics. In a feasibility study, we propose the instrumentation of a LDM that can measure the mass and speed of dust particles with a large detection area of $0.04 \mathrm{~m}^{2}$. With this LDM, we aim at quantitatively studying dust particles around the Moon, inclusive of interplanetary dust, $\beta$ meteoroids, interstellar dust, and possibly lunar dust that originate from the surface materials of the Moon. In this paper, we summarize the significance of dust particles around the Moon and report an overview of our instrument proposed for the next Japanese lunar mission SELENE-2.
\end{abstract}

Key words: Cosmic dust, interplanetary dust, interstellar dust, lunar dust, impact ionization detector, lunar exploration.

\section{Introduction}

Dust particles (or micrometeoroids) around the Moon, which we call lunar ambient dust particles, include interplanetary dust (IPD), $\beta$ meteoroids, interstellar dust (ISD), and possibly lunar dust that originates from the surface materials of the Moon. According to a direct measurement of the accretion rate to the Earth by Love and Brownlee (1993), several thousands of tons of dust particles may fall onto the Moon every year, supplying materials to its surface layer. When the inflow dust particles hit the surface of the Moon, or any other airless body (Mercury, asteroids, etc.), the resulting melting and vaporization redden materials on the surface. Therefore, the reflectance spectrum of

Copyright (c) The Society of Geomagnetism and Earth, Planetary and Space Sciences (SGEPSS); The Seismological Society of Japan; The Volcanological Society of Japan; The Geodetic Society of Japan; The Japanese Society for Planetary Sciences; TERRAPUB.

doi: $10.5047 /$ eps.2011.05.038 lunar surface materials can be changed from their primordial characteristics by the hypervelocity impact of dust particles because of the space weathering (Hapke et al., 1975; Keller and McKay, 1993; Sasaki and Hiroi, 2008). This change might cause a problem in estimating mineralogical composition from the reflectance spectrum of the Moon. The quantitative relation between dust flux and reflectance change is uncertain since the flux of the inflow dust colliding with the lunar surface has not yet been quantitatively estimated for different dust sizes. Therefore, an in-situ measurement of "inflow" dust particles is very important for an understanding of the material composition of the lunar surface. The regolith on the lunar surface may be accelerated to energetic lunar dust by some mechanisms. As a result, some lunar material possibly leaves the gravitational influence of the Moon (like lunar meteorites). Although the escaping mass flux is still uncertain, primordial surface materials might be exposed by such a mass-loss mechanism from 
Table 1. Properties of dust particles around the Moon.

\begin{tabular}{|c|c|c|c|}
\hline \multirow{2}{*}{ Dust } & \multirow{2}{*}{ Origin } & \multicolumn{2}{|c|}{ Kinetic property in heliocentric coordinates } \\
\hline & & Velocity (mass) & Direction of arrival \\
\hline Interstellar dust & Local interstellar cloud & $25 \mathrm{~km} / \mathrm{s}^{*}\left(<10^{-8} \mathrm{~g}^{*}\right)$ & $\begin{array}{l}\text { Ecliptic longitude of } 252^{\circ} \& \text { ecliptic lati- } \\
\text { tude of } 5^{\circ *}\end{array}$ \\
\hline Interplanetary dust & $\begin{array}{l}\text { Cometary and asteroidal ma- } \\
\text { terials }\end{array}$ & $<42 \mathrm{~km} / \mathrm{s}^{* *}\left(<10^{-5} \mathrm{~g}^{* *}\right)$ & $\begin{array}{l}\text { Anti-apex of the Earth or higher eccentric- } \\
\text { ities and various inclinations }\end{array}$ \\
\hline$\beta$ meteoroids & $\begin{array}{l}\text { Interplanetary dust swept by } \\
\text { solar radiation }\end{array}$ & $>42 \mathrm{~km} / \mathrm{s}^{* * *}\left(<10^{-12} \mathrm{~g}^{* * * *}\right)$ & Solar direction \\
\hline Lunar dust & $\begin{array}{l}\text { Materials on the lunar sur- } \\
\text { face, regolith }\end{array}$ & $>0 \mathrm{~km} / \mathrm{s}^{* * * * *}$ (unknown) & Lunar direction \\
\hline
\end{tabular}

* Refer to Kimura et al. (2003a, b).

**Refer to Fechtig (1982) and Grün et al. (1985).

***Escape velocity at the Earth/Moon location with respect to the Sun's gravity.

**** The mass of which a spherical particle with a density of $2 \mathrm{~g} / \mathrm{cm}^{3}$ has a high $\beta$ factor greater than 0.5 . Such dust particles can be accelerated by radiation pressure to above the escape velocity at $1 \mathrm{AU}$ (Zook and Berg, 1975).

***** Selenocentric velocity measured in an orbit of $100-\mathrm{km}$ altitude.

the lunar surface. Thus, the "outflow" dust particles are another important factor to understand the chemical composition of material of the lunar surface. There have been several past missions for dust observation around the Moon, but we need further data to improve the statistics for a quantitative study of lunar ambient dust particles; in particular, the outflow dust particles. The next Japanese lunar mission SELENE-2, after the successful mission Kaguya (formerly known as SELENE), is planned to be launched in the mid 2010 s and to consist of a lander, a rover, and an orbiter, as a transmitting satellite to the Earth (Matsumoto et al., 2008). Here, we propose a cosmic-dust particle detector LDM (Lunar Dust Monitor) to be onboard the orbiter. In a lunar orbit that is far further from the Earth and, in particular, the earth's geosynchronous orbit, cosmic-dust observation takes place in a clean environment, which is not influenced by a background of man-made space debris. As for dust observation in a debris-free environment for one year, cosmicdust observation with a large detection area is certainly of significance. Recently, large-detection-area dust counters have been proposed for future space missions in Earth orbit (Sternovsky et al., 2007; Grün et al., 2009). If those missions are realized, many subjects that have been neglected due to insufficient statistics of dust observation might be solved. The detection area of the LDM proposed here is smaller than those mentioned above, but still has larger detection area than those used in past dust missions in lunar orbit. In this paper, we summarize the observational significance of lunar ambient dust and present an overview of our instrument proposed to the SELENE-2 mission, which has a large detection area thereby enabling a significant amount of data to be obtained.

\section{Significance of the Observation of Lunar Am- bient Dust Particles}

\subsection{Lunar ambient dust particles}

Hiten and Nozomi observed dust particles and measured their fluxes in lunar orbit (Iglseder et al., 1996; Sasaki et al., 2002, 2007). Hiten orbited the Moon from February 1992 to April 1993, and the dust counter MDC (Munich Dust Counter), which was the only science payload of Hiten, detected about 150 dust particles in lunar orbit. Nozomi was a Japanese Mars explorer; the Nozomi-MDC (Mars Dust Counter) observed dust particles for five months in the Earth's gravitational sphere before leaving for Mars. Nozomi made only flybys of the Moon without entering lunar orbit. These observations by Hiten- and Nozomi-MDCs obtained a small dataset of dust particles for statistics, excluding earth-orbiting dust, once in a week, because the dust detectors had small sensitive areas, $0.01 \mathrm{~m}^{2}$ and $0.014 \mathrm{~m}^{2}$, respectively. Dust particles around the Moon should be classified on the basis of their origins: ISD, IPD, $\beta$ meteoroids, and possibly dust particles that have originated from the Moon. Table 1 summarizes a rough criterion for the identification of dust particles around the Moon. They can be inferred from their kinematic properties: the velocities and the arrival directions. If the population of dust components around the Moon is determined by observation, we can increase our knowledge of the contribution of inflow and outflow dust particles to lunar surface materials.

2.1.1 Source of supply of the Moon's volatile materials Materials on the lunar surface contain volatiles, as evidenced by a sodium atmosphere on the Moon. The volatiles should already be exhausted from the surface layer on the Moon if they are supplied only from the lunar interior because the diffusion of sodium on the Moon is inadequate to account for the observed atmospheres (Killen, 1989; Sprague, 1990; Stern, 1999; Sarantos et al., 2008). The sodium atmosphere, however, still remains if cometary dust is one of the supply sources. A past observation shows that the sodium atmosphere of the Moon changes in synchronization with Leonids (Smith et al., 1999; Shiokawa et al., 2000). Furthermore, cometary dust may be a contributing source of water ice in the pole regions of the Moon (Berezhnoy et al., 2003). A dust detector onboard a spacecraft in lunar orbit can obtain data that are necessary to infer the origin of volatiles on the Moon.

2.1.2 Evolution of the lunar regolith Dust particles play an important role in the space weathering of airless bodies such as the Moon. Bombardment of high-velocity dust particles of the lunar surface contributes to the production of its regolith layer. As we have mentioned in Introduction, space weathering affects its optical properties. As the surface undergoes space weathering, the overall 


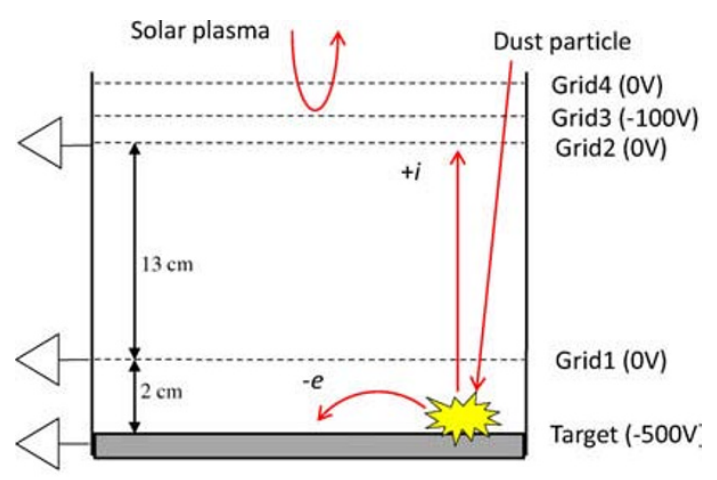

LDM1009-01

Fig. 1. Schematic of the cross-sectional view of LDM-S, the sensor part of the LDM.

albedo is reduced, reflectance decreases with wavelength, and the depth of its diagnostic absorption bands is reduced. The mineral compositions of lunar materials derived from remote-sensing observations of the reflectance spectrum become ambiguous due to the space-weathering effect. Since the bombardment rate of dust particles of the Moon has not been measured precisely, any changes in the reflectance of lunar surface materials have not been evaluated quantitatively. It is important to measure the current flux of inflow dust particles, from which the weathering effect in the past can be inferred. The latitudinal distribution of the inflow position of dust particles is also interesting, because the latitude dependence of space weathering may reveal a leading cause of space weathering.

2.1.3 Ejecta escaped from the Moon's gravitational sphere Surface materials might escape from the Moon, although they have not yet been directly observed. Small bodies such as satellites and asteroids have weak gravitational fields that enable surface materials to easily escape as impact ejecta of meteoroid bombardment. The dust detector subsystem (DDS) onboard Galileo detected dust particles ejected from the Jovian satellites, Ganymede, Europa, and Callisto. A theoretical estimate of the total mass of impact ejecta is difficult because of unknown ejection mechanisms. However, observation by a dust detector with a large detection area may determine the total mass of outflow materials. Small ejecta from the Moon, of a size assumed to be less than $1 \mu \mathrm{m}$, may be related to levitation dust observed by Apollo astronauts (McCoy, 1976; Zook and McCoy, 1991). It is expected that the grain size of levitation dust at a certain position in the lunar orbit depends on the grain size of regolith materials at the nadir.

2.1.4 Inflow dust related to meteor streams Recent studies suggest that the flux of inflow dust increases when meteor streams appear (see, e.g., Bruno, 2007). An observation over more than one year may verify this hypothesis by examining the variation of the dust flux with meteor streams.

\section{Instrumentation of the Lunar Dust Monitor 3.1 Principle of measurement}

As shown in Fig. 1, LDM-S, that is the sensor part of LDM, has four meshed grids (Grid1, Grid2, Grid3, and

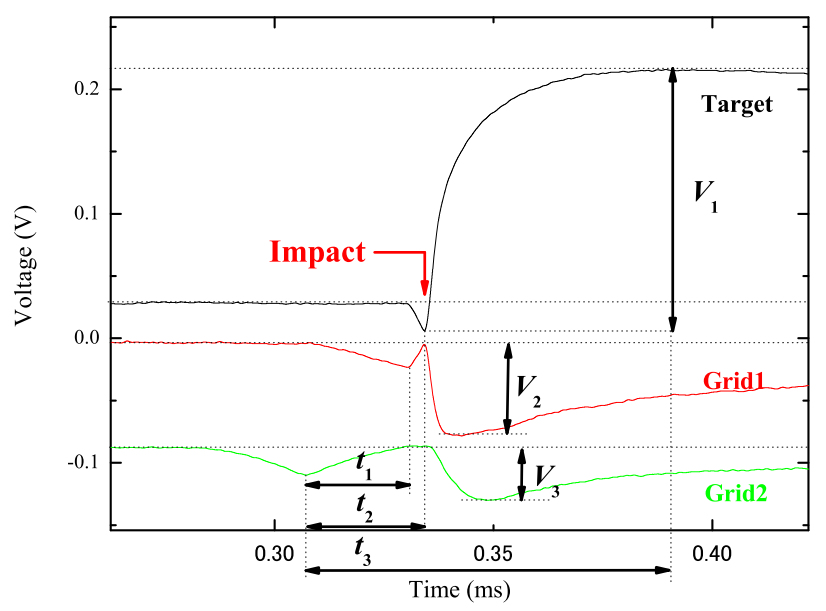

Fig. 2. Example of a typical event measured with a bread-board model in the laboratory.

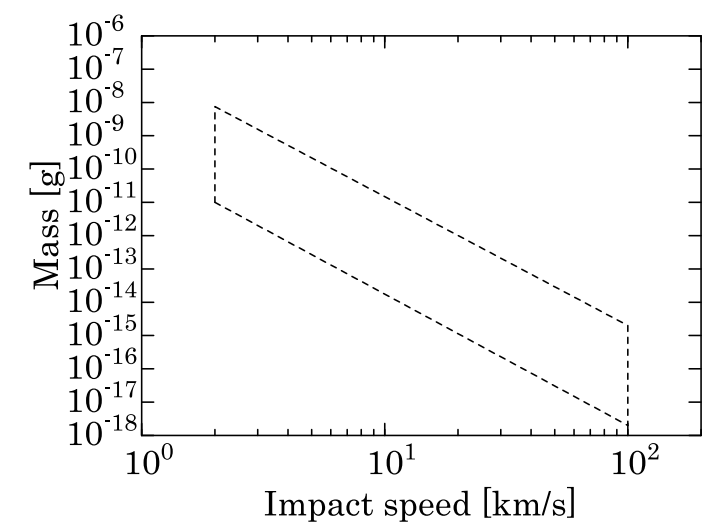

Fig. 3. The sensitivity of the LDM is shown by an area surrounded by a dashed line.

Grid4) parallel to the target whose effective area is $0.04 \mathrm{~m}^{2}$ $(20 \mathrm{~cm} \times 20 \mathrm{~cm})$. The grids are $90 \%$ transparent: Grid1 is $2 \mathrm{~cm}$ apart from the target and Grid 2 is $15 \mathrm{~cm}$ from the target. Two meshed grids, Grid3 and Grid4, between which a negative-bias high voltage of $-100 \mathrm{~V}$ is applied to reflect the solar-wind electrons, were added. LDM-S has a large target (gold-plated $\mathrm{Al}$ ), to which a high voltage of $+500 \mathrm{~V}$ is applied. Grid1, Grid2, and the target, are individually followed by charge-sensitive amplifiers, which convert charge signals induced by electrons and ions to voltage signals that are fed to a following flash ADC driven with $10 \mathrm{MHz}$. Assuming the surface potential of $+5 \mathrm{~V}$ for incident dust particles (Mukai et al., 2001), the lower detection limit of dust size is about $2 \mu \mathrm{m}$ in radius. Figure 2 shows an example of the waveforms of a typical event measured with a breadboard model in a laboratory. The waveforms from Grid1, Grid2, and the target, can be stored and be sent back to ground. We can deduce the mass and speed information of impacting dust particles from the recorded waveforms. This impact ionization type detector is designed to detect charged dust particles and to measure the kinematic parameters, mass and speed. The speed of a dust particle is obtained by measuring the time of flight (TOF) between Grid1 and Grid2. When the charged dust particle passes through Grid2 and Grid1, it induces an electric signal on the grids. 
Table 2. Measuring capability of LDM.

\begin{tabular}{|l|l|l|l|}
\hline \multicolumn{2}{|c|}{} & Science requirement & Performance target \\
\hline \hline \multirow{2}{*}{ Velocity } & Range & $1-100 \mathrm{~km} / \mathrm{s}$ & $0.1-100 \mathrm{~km} / \mathrm{s}$ \\
\cline { 2 - 4 } & Precision & better than $30 \%$ & better than $30 \%$ \\
\hline \multirow{3}{*}{ Mass } & Range & $\begin{array}{l}10^{-14}-10^{-11} \mathrm{~g} \\
(@ 10 \mathrm{~km} / \mathrm{s})\end{array}$ & $\begin{array}{l}\text { as shown in Fig. } 3 \\
10^{-14}-10^{-11} \mathrm{~g}(@ 10 \mathrm{~km} / \mathrm{s})\end{array}$ \\
\cline { 2 - 4 } & Precision & better than an order of magnitude & better than an order of magnitude \\
\hline
\end{tabular}

As shown in Fig. 2, the signals from Grid1 and Grid2 show negative peaks before the moment of the impact. Those signals have peaks corresponding to the time that the incident charged dust particle pass through the grids and this corresponds to "start" and "stop" of TOF. Time separation between the peaks shows a TOF of the incident dust particle between the grids. The speed of the incident dust particle is derived from the TOF and the distance between the grids. Measuring the time separation, we can determine the speed of the particle. Uncertainty in the speed determination cannot be completely erased, because the path length between the grids depends on the incident angle. We obtain the mass of the incident dust particle by observing the impact plasma generated from the impact of the hypervelocity dust particle on the target plate. The quantity of the impact plasma is obtained by reading the pulse height of the signal from the target plate, V1, shown in Fig. 2. When the incident particle with hypervelocity impacts on the target, the impact generates a plasma gas of electrons and ions. The electrons of the plasma are collected by the target and the ions are accelerated toward Grid1 by the electric field. Some of the ions drift through Grid1 and reach Grid2. The mass of the incident dust particle is a function of the quantity and drift time of the impact plasma as follows;

$$
\begin{aligned}
t_{\mathrm{r}} & =c_{\mathrm{g}} \cdot v^{\alpha}, \\
Q / m & =c_{\mathrm{r}} \cdot v^{\beta},
\end{aligned}
$$

where $t_{\mathrm{r}}$ is the rising time of the target signal, $Q$ is the charge collected on the target, $m$ and $v$ are the mass and the speed of the incident dust particle, respectively (Friichtenicht and Slattery, 1963; Auer and Sitte, 1968). The parameters $c_{\mathrm{g}}, c_{\mathrm{r}}, \alpha, \beta$, have been determined by calibration experiments using a van de Graaff accelerator at the Max Planck Institute for Nuclear Physics in Heidelberg: $c_{\mathrm{g}}=7.6 \times 10, c_{\mathrm{r}}=5.50 \times 10^{-5}, \alpha=-1.44$, $\beta=3.91$. The LDM detects dust particles with a sensitivity as shown in Fig. 3. Using a bread-board model of the LDM, we are evaluating the conceptual design of the LDM described above and conducting experiments to optimize its performance (Ohashi et al., 2010). From the results of experiments accounting for the uncertainties of the parameters and the field of view (FOV) of the instrument, the speeds of incident dust particles larger than $2 \mu \mathrm{m}$ can be measured as low as $2 \mathrm{~km} / \mathrm{s}$ within an uncertainty of about $30 \%$ owing to their TOFs between the grids. For incident dust particles smaller than $2 \mu \mathrm{m}$, the uncertainties of the mass and the speed are an order of magnitude and about $70 \%$, respectively. The assumed measuring capability of the LDM is tabulated in Table 2. Some of the incident particles can impact on the side wall of the impact ionization detector, a so- called side-wall impact event (Willis et al., 2004; Altobelli et al., 2004). The LDM will also have such side-wall impact events in orbit and the observed data has to be corrected according to a secondary impacts experiment, such as that of Willis et al. (2004).

\subsection{Evaluation of the detection area of the LDM}

We propose a dust monitor that is operated in lunar orbit, called the lunar dust monitor (LDM). The detection area of the sensor part is $0.04 \mathrm{~m}^{2}$ (rectangle, $20 \mathrm{~cm} \times 20 \mathrm{~cm}$ ) and the FOV is $\pm 62^{\circ}$. This instrument aims to observe several hundred counts of dust particles, the origins of which are well identified. The detection area of the LDM has been constrained from the following arguments: A dust detector called MDC onboard Hiten that was launched on January 24th, 1990, observed dust particles in lunar orbit. The Hiten-MDC, of which the detection area was $0.010 \mathrm{~m}^{2}$, detected 150 counts of dust particles during 14 months of operation, from February 15, 1992, through April 10th, 1993, (Iglseder et al., 1996). The Hiten-MDC observed dust particles with velocities from $1.8 \mathrm{~km} / \mathrm{s}$ up to over $70 \mathrm{~km} / \mathrm{s}$ and masses between $10^{-7} \mathrm{~g}$ and $10^{-16} \mathrm{~g}$. Using the same procedure as described above, the mass and velocity of the impacted dust particles can be derived from the Hiten-MDC observation data. Our LDM has a geometric configuration of sensor and dynamic ranges of mass and velocity for impacted dust particles similar to the Hiten-MDC. The Hiten-MDC had a single grid as a top layer while the LDM has four grids that have $90 \%$ transparencies, individually. Therefore, only about $65 \%$ dust particles with incident angles within the FOV can pass through the grids and reach the target. The FOV of the LDM, $\pm 62^{\circ}$, should also be taken account. As a scientific requirement, it is preferable to detect several hundred counts of dust particles during the mission period (assumed one year) in order to identify their origins (meteoroids, comets, and interstellar matter) by the orbits of the observed dust particles. Therefore, the LDM needs a detection area several times larger than those missions and thus the detection area was specified to be $0.04 \mathrm{~m}^{2}$. The LDM aims to observe the dust environment in lunar orbit and dust components shown in Table 1 are expected to be detected. Those various dust components have different flux and arrival directions. To detect them evenly, the mounting position and orientation of the LDM should be carefully considered, taking into account the flux and arrival direction of the dust components.

\section{Collaboration with the Preceding Mission of LADEE-LDEX}

The Lunar Dust EXperiment (LDEX) onboard LADEE, which will be launched in 2012, is designed to map the spa- 
tial and temporal variability of the dust size and density distributions in the lunar environment (Horányi et al., 2009). LDEX will observe the lunar environment for about 90 days at $50-\mathrm{km}$ altitude in a nominal case. It has a sensor area of $0.01 \mathrm{~m}^{2}$. The observation data of LDEX in the low orbit will determine the dust outflow rate from the Moon accurately while SELENE-2 will orbit at 100-km altitude and can detect the more energetic outflow dust particles. Combining the observation results of LDEX and LDM will improve our knowledge of the conventional model of the interplanetary flux at $1 \mathrm{AU}$ and the lunar flux proposed by Grün et al. (1985).

\section{Concluding Remarks}

In this paper, we propose a future observation plan of dust particles in lunar orbit onboard the orbiter of the SELENE-2 mission. The proposed instrumentation has a larger detection area $\left(0.04 \mathrm{~m}^{2}\right)$ than that employed in past lunar missions which will enable it to obtain enough statistics of ambient dust particles for a quantitative study to determine the origin of the dust particles accurately. The orbiter of SELENE-2 may be planned to be in operation for one year or longer, and the LDM is expected to observe more than 100 counts of dust particles with enough kinematic information to identify their origins in lunar orbit. It has been observed that the Sun is at an extremely low activity level at the time of writing this paper. Under such circumstances, more ISD, the orbit of which is less affected by the interplanetary magnetic field, might be observed at $1 \mathrm{AU}$.

Acknowledgments. We would like to give heartful thanks to Mr. S. Bugiel who operates the Van de Graaf accelerater of the Heidelberg Dust Accelerator Facility in MPI-K and also to Mr. T. Omata who operates the Van de Graaf accelerator of HIT in the University of Tokyo, for LDM calibration. Special thanks also go to $M$. Horányi and an anonymous reviewer whose opinions and information have helped us very much throughout the production of this paper. We also gratefully appreciate the financial support of the Exchange Program of CPS that made it possible to complete our paper. Finally, this research was partially supported by Grant-inAid for Scientific Research (B), No. 21340040.

\section{References}

Altobelli, N., H. Krüger, R. Moissl, M. Landgraf, and E. Grün, Influence of wall impacts on the Ulysses dust detector on understanding the interstellar dust flux, Planet. Space Sci., 52, 1287-1295, 2004.

Auer, S. and K. Sitte, Detection technique for micrometeoroids using impact ionization, Earth Planet. Sci. Lett., 4, 178-183, 1968.

Berezhnoy, A . A., N. Hasebe, T. Hiramoto, and B. A. Klumov, Possibility of the Presence of $\mathrm{S}, \mathrm{SO}_{2}$, and $\mathrm{CO}_{2}$ at the Poles of the Moon, Astron. Soc. Jpn., 55, 859-870, 2003.

Bruno, M., G. Cremonese, and S. Marchi, Neutral sodium atoms release from the surfaces of the Moon and Mercury induced by meteoroid impacts, Planet. Space Sci., 55, 1494-1501, 2007.

Fechtig, H., in Comets, edited by Wilkening, L. L., pp. 370-382, University of Arizona Press, Tucson, 1982.

Friichtenicht, J. F. and J. C. Slattery, Ionization associated with hypervelocity impact, NASA TN D-2091, 1963.

Grün, E., H. A. Zook, H. Fechtig, and R. H. Giese, Collisional balance of the meteoritic complex, Icarus, 62, 244-272, 1985.

Grün, E., R. Srama, N. Altobelli, K. Altwegg, J. Carpenter, L. Colangeli, K.-H. Glassmeier, S. Helfert, H. Henkel, M. Horányi, A. Jäckel, S. Kempf, M. Landgraf, N. McBride, G. Moragas-Klostermeyer, P. Palumbo, H. Scholten, A. Srowig, Z. Sternovsky, and X. Vo, DuneXpress, Exp. Astron., 23(3), 981-999, 2009.

Hapke, B., W. Cassidy, and E. Wells, Effects of vapor-phase deposition processes on the optical, chemical, and magnetic properties of the lunar regolith, The Moon, 13, 339-353, 1975.

Horányi, M., Z. Sternovsky, E. Gruen, R. Srama, M. Lankton, and D. Gathright, The Lunar Dust EXperiment (LDEX) on the Lunar Atmosphere and Dust Environment Explorer (LADEE) Mission, 40th LPSC, Abstract \#1741, 2009.

Iglseder, H., K. Uesugi, and H. Svedhem, Cosmic dust measurements in lunar orbit, Adv. Space Res., 17, 177-182, 1996.

Keller, L. P. and D. S. McKay, Discovery of vapor deposits in the lunar regolith, Science, 261, 1305-1307, 1993.

Killen, R. M., Crustal diffusion of gases out of Mercury and the Moon, Geophys. Res. Lett., 16(2), 171-174, 1989.

Kimura, H., I. Mann, and E. K. Jessberger, Elemental abundances and mass densities of dust and gas in the Local Interstellar Cloud, Astrophys. J., 582, 846-858, 2003a.

Kimura, H., I. Mann, and E. K. Jessberger, Composition, structure, and size distribution of dust in the Local Interstellar Cloud, Astrophys. J., 583, 314-321, 2003b.

Love, S. G. and D. E. Brownlee, A direct measurement of the terrestrial mass accretion rate of cosmic dust, Science, 262(5133), 550-553, 1993.

Matsumoto, K., T. Hashimoto, T. Hoshino, S. Tanaka, M. Otsuki, and J. Kawaguchi, Japanese 1st Moon Lander SELENE-2 as SELENE FollowOn, Joint Annual Meeting of LEAG-ICEUM-SRR, LPI Contribution No. 1446, 86, 2008.

McCoy, J. E., Additional photometric studies of light scattering above the lunar terminator from Apollo orbital corona photography, Abstr. Lunar Planet. Sci. Conf., 7, 515, 1976.

Mukai, T., J. Blum, A. M. Nakamura, R. E. Johnson, and O. Havenes, Physical processes on interplanetary dust, in Interplanetary Dust, edited by Grün et al., p. 445, Springer, 2001.

Ohashi, H., M. Kobayashi, S. Sasaki, H. Shibata, M. Fujii, K. Nogami, T. Iwai, H. Kimura, M. H. Nakamura, T. Hirai, E. Gruen, and R. Srama, Lunar dust monitor to be onboard next Japanese lunar mission SELENE-2, 38th COSPAR, 18-25 July 2010 in Bremen, 2010.

Sarantos, M., R. M. Killen, A. S. Sharma, and J. A. Slavin, Influence of plasma ions on source rates for the lunar exosphere during passage through the Earth's magnetosphere, Geophys. Res. Lett., 35, L04105, 2008.

Sasaki, S. and T. Hiroi, How does space weathering depend on the surface condition of airless bodies (asteroids, the Moon, and Mercury)?, 39th LPSC, Abstract\#1625, 2008.

Sasaki, S., E. Igenbergs, H. Ohashi, R. Münzenmayer, W. Naumann, G. Hofschuster, M. Born, G. Farbar, F. Fischer, A. Fujiwara, A. Glasmacher, E. Grün, Y. Hamabe, H. Iglseder, T. Kawamura, H. Miyamoto, K. Morishige, T. Mukai, T. Naoi, K. Nogami, G. Schwehm, and H. Svedhem, Observation of interplanetary and interstellar dust particles by Mars Dust Counter (MDC) on board NOZOMI, Adv. Space Res., 29, 1145-1153, 2002.

Sasaki, S., E. Igenbergs, H. Ohashi, R. Senger, R. Münzenmayer, W. Naumann, E. Grün, K. Nogami, I. Mann, and H. Svedhem, Summary of interplanetary and interstellar dust observation by Mars Dust Counter on board NOZOMI, Adv. Space Res., 39, 485-488, 2007.

Shiokawa, K., M. K. Ejiri, T. Ogawa, and T. Nakamura, Distant lunar sodium tail observed in the Japanese local-time sector during the Leonid meteor shower of 1998, J. Geophys. Res., 105, 24621-24626, 2000.

Smith, S. M., J. K. Wilson, J. Baumgardner, and M. Mendillo, Discovery of the distant lunar sodium tail and its enhancement following the Leonid meteor shower of 1998, Geophys. Res. Lett., 26, 1649-1652, 1999.

Sprague, A. L., A diffusion source for sodium and potassium in the atmospheres of Mercury and the Moon, Icarus, 84, 93-105, 1990.

Stern, A. S., The lunar atmosphere: History, status, current problems, and context, Rev. Geophys., 37, 453-491, 1999.

Sternovsky, Z., K. Amyx, G. Bano, M. Landgraf, M. Horányi, S. Knappmiller, S. Robertson, E. Grün, R. Srama, and S. Auer, Large area mass analyzer instrument for the chemical analysis of interstellar dust particles, Rev. Sci. Instr., 78(1), 014501-014501-10, 2007.

Willis, M. J., M. J. Burchell, M. J. Cole, and J. A. M. McDonnell, Influence of impact ionisation detection methods on determination of dust particle flux in space, Planet. Space Sci., 52, 711-725, 2004.

Zook, H. A. and O. E. Berg, A source for hyperbolic cosmic dust particles, Planet. Space Sci., 23, 183-203, 1975.

Zook, H. A. and J. E. McCoy, Large scale lunar horizon glow and a high altitude lunar dust exosphere, Geophys. Res. Lett., 18, 2117-2120, 1991.

M. Kobayashi (e-mail: kobayashi.masanori@perc.it-chiba.ac.jp), H. Ohashi, S. Sasaki, H. Shibata, T. Iwai, M. Fujii, K.-i. Nogami, H. Kimura, M. H. Nakamura, T. Hirai, R. Srama, and E. Grün 